\title{
Semantic similarity and the A-B, C-D paradigm in STM
}

H. C. A. DALE

DEPARTMENT OF PSYCHOLOGY, UNIVERSITY OF HULL, ENGLAND

Although $R I$ is influenced by the semantic similarity of stimuli with the $A-B, C-D$ paradigm in normal $P-A$ learning, comparable effects have not been found in STM. Earlier work was open to the criticism that the normal RI procedure was not paralleled by the STM conditions. The present experiments rectify this deficiency, but the effects of stimulus similarity are still absent.

Baddeley \& Dale (1966) compared the effect of stimulus similarity upon retroactive inhibition (RI) in long- and short-term memory using the A-B, C-D paradigm. They employed bisyllabic adjectives and found that when the original list (OL) and interpolated list (IL) stimuli were paired so that members of the pairs were semantically similar, i.e., were synonomous, retention was poor compared with the control condition in which "A" and "C" stimull were unrelated. No comparable effect was obtained in STM, however.

For their STM experiments a modification of Murdoch's (1963) technique was used. (Murdoch presented each list once and tested only one pair, whereas Baddeley and Dale tested all pairs.) In analyzing for interference effects it was assumed that the single presentation of the list corresponded to both OL and IL phases of the classical RI procedure. Thus if two pairs were presented and retention of the first was measured, it was assumed that the second pair corresponded to the IL phase. The correspondence is not exact, however. In the classical procedure, ol learning is tested before IL presentation, and $S$ is not led to expect a retention test after IL. Furthermore, it has been demonstrated by Lester (1932) and by Postman \& Stark (1962) that if the normal instructions are modified by warning $S$ that a retention test of $O L$ will be given after IL, then the disruptive influence of the IL is considerably reduced.

The present experiments attempt to test the effect of stimulus similarity in PA learning over the short periods normally encompassed in work on STM while preserving the normal procedure.

\section{EXPERIMENT 1}

\section{Method}

Each S learned two lists, each consisting of three palrs. The OL was presented once by being read aloud by $E$ at a rate of one word per sec with 2 sec between pairs. After $10 \mathrm{sec}$, retention of all three pairs was tested in an unpredictable order allowing $4 \mathrm{sec}$ for each response. After another $10 \mathrm{sec}$ interval, the IL was presented and tested at the same rate. Finally, retention of OL was demanded $10 \mathrm{sec}$ after the IL test. In all retention tests the stimulus word was read aloud once by $E$ at the beginning of the $4 \mathrm{sec}$ period.

The material was taken from the LTM experiment of Baddeley and Dale. They used lists of eight pairs. To provide a representative test the present experiment was replicated on two different samples of their material so that six pairs were taken from each of their lists of eight.

The experimental design was copied from the Baddeley and Dale study. Four conditions were run: $A-B, A-Y$, $X-B$, and $X-Y$, where synonomous pairs of Ss occurred in $A$ and $B$ lists and also in $X$ and $Y$ lists. As in standard PA procedures, orders of presentation and test were different. Since the time that Items have to be remembered is critical in STM experimentation, procisely the same relation between presentation and test orders was used in all conditions. Since two samples of material were employed, eight groups were required. The size of each group made avallable for testing was not under the control of the Es, and it varied from 23 to 30 . All Ss were young, newly enllsted men. Each was used in only one condition.

\section{Results}

Ss who scored zero on OL or IL were rejected. Mean retest scores of those remaining are shown in Table 1 , where they are categorized according the the degree of OL retention achieved. In general, retention was slightly better with different IL material. Reference to the table shows this is due entirely to those who had two correct OL Items. Separate analysis of these data show the effect does not approach statistical significance $\left(X^{2}=1.3, d f=1\right)$.

\section{EXPERIMENT 2}

Since the single trial experiment failed to show any significant effect of similarity, it was repeated with two OL trials and two IL trials.

Method

The material was precisely the same as that of Experiment 1. The second learning trial, using a new order of presentation, was given $10 \mathrm{sec}$ after the first. Fresh Ss from the same source were employed. The sizes of groups tested varied from 14 to 21 .

Table 1 Mean retest scores in Experiment 1

\begin{tabular}{lllccc}
\hline & OL & 1 & 2 & 3 & $\Sigma$ \\
\hline Similar IL & $\overline{\mathrm{x}}$ & 0.58 & 0.75 & 1.50 & 0.84 \\
(A-B+X-Y) & $\mathrm{N}$ & 36 & 12 & 16 & 64 \\
Different IL & $\overline{\mathrm{X}}$ & 0.50 & 1.47 & 1.45 & 1.02 \\
(A-Y+X-B) & $\mathrm{N}$ & 30 & 15 & 19 & 64 \\
\hline
\end{tabular}


Table 2 Mean retest scores in Experiment 2

\begin{tabular}{lccccc}
\hline & $\begin{array}{c}\text { OL } \\
\text { (tr. 2) }\end{array}$ & 1 & 2 & 3 & $\Sigma$ \\
\hline Similar IL & $\bar{x}$ & 0.77 & 0.20 & 1.20 & 0.98 \\
(A-B+X-Y) & $\mathrm{N}$ & 13 & 5 & 30 & 48 \\
Different IL & $\overline{\mathrm{X}}$ & 0.54 & 0.48 & 1.17 & 0.87 \\
(A-Y+X-B) & $\mathrm{N}$ & 13 & 14 & 35 & 62 \\
\hline
\end{tabular}

\section{Results}

Table 2 shows the mean level of retention for each level of $O L$ as assessed on the second trial. As with Experiment 1, Ss scoring zero on OL or IL have been excluded. In general, retention was better with similar IL than with different. The data from those Ss who had two OL items correct, however, showed better retention with different IL, but not significantly so. When their data is combined with data from the Ss in Experiment 1 who had two correct OL items, the overall result is still not statistically significant $(p>0.10)$. DISCUSSION

We must conclude from these two experiments that even when the STM procedure exactly models the LTM procedure, comparable effects of semantic similarity cannot be found. With Experiment 1, the mean retention period per item was $62 \mathrm{sec}$. With Experiment 2, it was $106 \mathrm{sec}$. These experiments can therefore be criticized for demanding retention over periods greater than those usually employed in STM studies. But this makes it even more surprising that the effects found in LTM are not present. Reference to the LTM condition of Baddeley and Dale shows that when the list length is increased to eight pairs and the number of trials of both $O L$ and IL to eight, then the disruptive effect of semantically similar stimuli can be demonstrated. The minimum number of pairs or trials required for these effects must be found by patient exploration. But it must be noted that when the PA procedure is abandoned, differential effects of semantic similarity upon RI in STM are obtained (Dale \& Gregory, 1966). Which is all rather puzzling.

\section{References}

BADDELEY, A. D., \& DALE, H. C. A. The effect of semantic similarity on retroactive interference in long and short-term memory. $J$. verbal Learn, verbal Behav., 1966, 5, 417-420.

DALE, H. C. A., \& GREGORY, M. Evidence of semantic coding in short-term memory. Psychon. Sci, 1966, 5, 75-76.

LESTER, O. P. Mental set in relation to retroactive interference. J. exp. Psychol, 1932, 15, 681-699.

MURDOCK, B. B. Jr. Short-term and memory paired associate learning. J. verbal Learn. verbal Behow., 1963, 2, 320-328.

POSTMAN, L., \& STARK, K. Retroactive interference as a function of set during the interpolated task. J. verbal Learn. verbal Behav., 1962, 1,304311 . 